\title{
Bebelos: spazio sacro, gradazioni di sacralità e gerarchie di partecipazione
}

Bebelos: Sacred Space, Sacrality Degrees, and Participation Hierarchies

Lucia Marrucci

\section{(2) OpenEdition}

\section{Journals}

\section{Edizione digitale}

URL: http://journals.openedition.org/mythos/435

DOI: $10.4000 /$ mythos. 435

ISSN: 2037-7746

\section{Editore}

Salvatore Sciascia Editore

\section{Edizione cartacea}

Data di pubblicazione: 1 décembre 2016

Paginazione: 159-171

ISBN: 978-88-8241-476-4

ISSN: $1972-2516$

\section{Notizia bibliografica digitale}

Lucia Marrucci, «Bebelos: spazio sacro, gradazioni di sacralità e gerarchie di partecipazione », Mythos [Online], 10 | 2016, online dal 24 septembre 2019, consultato il 28 septembre 2019. URL : http:// journals.openedition.org/mythos/435; DOI : 10.4000/mythos.435

Mythos 


\title{
Bebelos: spazio sacro, gradazioni di sacralità e gerarchie di partecipazione
}

\section{Lucia Marrucci}

Riassunto

Per accostarsi alle modalità con cui i Greci di età arcaica e classica pensavano il sacro e il rapporto tra uomini e dei, è utile l'analisi dei contesti nei quali ricorre l'aggettivo bebelos. Questa nozione, infatti, impossibile da ricondurre in modo assoluto al nostro concetto moderno di "profano", entra in rapporto con termini come hieros, hosios, hagnos e chiarisce l'importanza di processi fondamentali quali la creazione dello spazio sacro, il costituirsi della potenza divina, la costruzione di gerarchie di partecipazione al sacro.

\begin{abstract}
An analysis of contexts in which the word bebelos occurs is very useful in order to approach the way Greeks of archaic and classical ages used to consider the sacred as well as the relationship between men and gods. This idea is different from the modern notion of "profane" and it has relationships with words like hieròs, hosios, hagnos. Moreover, this idea is crucial in order to understand the creation of sacred space, the shaping of divine power and the construction of the sacred.
\end{abstract}

Parole chiave

Sacro - profano - spazio sacro - potenza divina - religione greca

Keywords

Sacred - Profane - Sacred space - Divine power - Greek religion

\begin{abstract}
$\mathrm{O}$ gni tentativo di accostamento alla nozione greca di "sacro" corrisponde alla necessità di misurarsi con una complessa varietà di significanti (hieros, hagnos, hosios, alsos, temenos, adyton, etc.) che rimandano, a loro volta, a una molteplicità di aspetti ancora più problematica: il rapporto tra uomini e dei, la creazione e l'essenza della potenza divina, le azioni compiute dagli uomini, tra le quali si distinguono, in particolare, gli atti costitutivi di uno spazio sacro.

Ma la difficoltà maggiore deriva dalla tentazione di applicare, in un simile percorso interpretativo, strumenti ermeneutici moderni, univoci e rigidi, inadatti a cogliere intrecci e sovrapposizioni, come, per esempio, l'antinomia tra "sacro" e "profano".

Con l'obiettivo di superare, almeno in parte, la rigidità di tale applicazione, cercheremo di analizzare come funziona una peculiare nozione di "profano", veicolata, per i parlanti greco di età arcaica e classica, dal termine bebelos. Ci soffermeremo pertanto sui contesti nei quali sono attivi la complementarietà tra bebelos e hieros, la competizione tra bebelos e hosios, la produttività di bebelos in rapporto alla costruzione dello spazio sacro e delle gerarchie in base alle quali gli uomini possono accedere e partecipare a tale spazio.
\end{abstract}




\section{Un modo greco di intendere il "profano"}

L'

opposizione moderna tra "sacro" e "profano", intesa come chiave per interpretare la modalità greca di pensare gli dei e i loro rapporti con gli uomini, mostra limiti chiari, riconosciuti, e ormai indiscutibilili ${ }^{1}$. Le argomentazioni contrarie alla possibilità del funzionamento lineare di un contrasto limpido tra le due categorie poggiano, di solito, sulla difficoltà di sovrapporre la nostra concezione di "sacro" ad una serie complessa ed eterogenea di nozioni greche, come quelle corrispondenti, per esempio, agli aggettivi hieros, hagnos, hagios e hosios 2 .

Nel denunciare le imperfezioni della moderna coppia antinomica, può tuttavia rivelarsi efficace anche una via di indagine alternativa, più marginale e per questo meno battuta, che si concentri non tanto sul primo elemento, il "sacro" (procedendo per accostamenti, tentativi fallimentari di sovrapposizioni e analogie), quanto piuttosto sul secondo, il "profano" (affidandosi alle potenzialità rivelatrici di scarti e opposizioni). Per comprendere alcuni aspetti delle modalità con cui i Greci di età arcaica e classica pensavano il "sacro", si può dunque provare a partire dalla consistenza, sia pure dubbia e articolata, di una nozione alla quale opporlo: quella, appunto, di "profano".

Lasciamo da parte la problematicità, centrale e per questo già molto studiata, di hosios ${ }^{3}$, inteso come termine chiamato a significare, sebbene in un modo parziale e condizionato da contesti specifici, quello che noi definiamo "profano". Esiste, infatti, un altro modo che i Greci hanno di designare quest'ultima nozione: per il tramite dell'aggettivo bebelos, attestato con frequenza assai minore rispetto ad hosios, eppure ugualmente dotato di una sua produttività.

La nozione corrispondente a bebelos sembrerebbe infatti implicare, nei contesti epigrafici e letterari nei quali ricorre, alcuni aspetti particolari -ed altrimenti inafferrabili - delle modalità con cui i Greci hanno pensato, agito e provato ad esprimere il rapporto con le potenze divine, aspetti non riconducibili, semplicemente, all'opposizione "sacro" / "profano". Nel sistema di nozioni elaborato dai parlanti greco per significare il sacro, bebelos si ritaglia pertanto una propria sfera di produttività non semplicemente a partire dalla relazione di antinomia e complementarietà che esso instaura con hieros, ma anche dai suoi rapporti con i sostantivi alsos, temenos, adyton.

Dall'analisi di queste relazioni emerge come bebelos, non in tutto sovrapponibile a hosios, come è ovvio, sembri tuttavia contendere a quest'ultimo la capacità di esprimere il punto di vista umano rispetto al sacro, in una prospettiva, però - questo è quello che cercheremo di dimostrare - diversa e particolare, perché connessa, in primo luogo, con l'agire dell'uomo nello spazio e, più nello specifico, con il movimento degli uomini rispetto allo spazio sacro.

Vedremo come i contesti nei quali si mostra attiva la produttività di bebelos coinvolgano una serie di ambiti fondamentali nel modo greco di pensare il "sacro", ambiti tra loro strettamente connessi: prima di tutto, (1) il processo stesso di costituzione dello spazio sacro da parte dell'uomo $^{4}$, il quale implica una delimitazione e insieme un rafforzamento della potenza divina (puissance) $^{5}$ che in esso agisce e risiede.

1 Sulla genesi, la storia e la produttività della coppia antinomica "sacro" / "profano" come strumento ermeneutico della storia delle religioni cf. BORGEAUd 1994; cf. inoltre BREMMER 1998.

2 Sul carattere complesso e plurale di queste nozioni cf. Di Donato 2001, 19-23. Più in generale: Parker 2011, 1-63.

3 Cf. Di Donato 2001, 19-23.

4 Sulle dinamiche di costituzione di uno spazio sacro e della ripartizione tra spazio umano e spazio divino, cf. Guettel Cole 2004, 30-66.

5 Sulla complessità del rapporto tra pensiero religioso e rappresentazione degli dei cf. VERNANT 1950 (= VERNANT 
Un meccanismo conseguente è poi (2) il determinarsi di una sorta di gradazione - tutt'altro che lineare - della sacralità di luoghi, di edifici e di oggetti, che si completa nel momento in cui (3) arriva a riflettersi sulle gerarchie di partecipazione al sacro concessa ai diversi gruppi umani, e sul carattere proporzionale del coinvolgimento con il sacro riconosciuto a ciascun individuo.

Bebela kai hiera

U no degli ambiti entro i quali l'aspetto del sacro implicato da bebelos si trova a funzionare è il sistema di opposizioni e complementarietà che lo legano a hieros e alla sfera degli hiera .

Il caso forse più esplicito non deriva dalla produzione letteraria, ma dalla celebre iscrizione sulla legge sacra di Cirene ${ }^{7}$, dove bebelos concorre a delineare, insieme a miaros e, appunto, a hieros, le tre distinte finalità cui è possibile destinare il legname proveniente da/cresciuto in un santuario ${ }^{8}$.

Ed è sempre sullo sfondo degli hiera, e della loro polivalenza, che bebelos viene messo a fuoco anche in un paio di testimonianze restituiteci dalla storiografia del V secolo.

L'espressione $\dot{\varepsilon} v \tau \tilde{\omega} \beta \varepsilon \beta \eta \dot{\lambda} \lambda \omega$ usata da Erodoto ${ }^{9}$ per descrivere il luogo in cui caddero i Persiani, messi in fuga dagli Spartani a Platea, serve a rendere più chiara e insieme più specifica l'indicazione che immediatamente la precede: peri to hiron. Lo spazio situato intorno al santuario, to hiron, e pertanto distinto da esso, trova traduzione e concretezza nell'aggettivo sostantivato to bebelon ${ }^{10}$. Oggetto di meraviglia e di stupore, il thoma che lo storico vuole condividere con il suo pubblico, quasi a stimolarne la reazione ${ }^{11}$, è proprio la qualità dello spazio ( $\dot{\varepsilon} \nu \tau \tilde{\omega}$ $\beta \varepsilon \beta \eta ́ \gamma(\omega)$ in cui si verifica la morte dei Persiani, i quali evitano di entrare nel bosco sacro e nel recinto sacro di Demetra, e cadono, invece, in un luogo la cui identificazione è il prodotto della sua diretta opposizione (o meglio: distinzione) rispetto a to hiron. Erodoto è assai chiaro circa il fatto che bebelos si riferisca qui a qualcosa di distinto da alsos e da temenos, ma la determinazione ultima del dato che egli intende consegnare ai propri destinatari è affidata, comunque, alla relazione con hieros: quello che non è hiron, perché posto intorno ad esso, è bebelon .

Meno diretta, ma non meno esplicita, è l’opposizione di cui si serve Tucidide, nel IV libro $^{12}$, per descrivere la trasgressione dei nomima ellenici compiuta da parte degli Ateniesi in occasione della campagna di Delio del 424. Anziché seguire la consuetudine istituzionalizzata (kathestekos) presso i Greci di astenersi dai templi (apechesthai ton hieron) durante gli attacchi, gli Ateniesi non si limitano a fortificare Delio e a stabilirvisi, ma si mettono a compiere lì (au-

1995, 191-195) Vernant 1965, 325-338, 339-351, Vernant 1975, 9-15; Vernant 1990, 17-28 della tr. it. (Roma 2003).

6 Cf. Di Donato 2001, Burkert 2003, 486 e Patera 2010, 541.

7 SEG IX, 72, ll. 10ss. Cf. Jeffrey 1961, 412 n. 76 e Rhodes, Osborne 2003, 494-505; sulla relazione tra hiaros, babalos e miaros nel contesto di questa iscrizione cf. ROBERTSON 20 Io, 284-5

8 Cf. Parker 1983, 165. Un altro caso di opposizione netta si trova nel testo del Corpus hippocraticum (Lex V, 2, cfr. infra) sul quale torneremo a concentrarci più avanti- dove l'aggettivo ha tuttavia compiuto un notevole slittamento semantico ed è attribuito a persone.

9 Hdt. IX, 65, 2.

10 Cf. Burkert 2003, 198.

11 Sulla nozione di thoma e la sua funzione entro il sistema narrativo delle Storie erodotee cf. VIGNOLO MUNSON 2001, 232-265.

12 Thuc. IV, 97, 3.4. 
tothi) tutto ciò che gli uomini di solito fanno altrove (come, per esempio, attingere un'acqua intoccabile), in luoghi definibili, appunto, tramite l'aggettivo bebelos ${ }^{13}$. En bebeloi è, infatti, l'espressione utilizzata per segnalare il sovvertimento dei nomima, concepito come netto contrasto rispetto alle pratiche che, di norma, avrebbero dovuto svolgersi, o meglio non svolgersi, in rapporto agli hiera. I Greci di Atene, in effetti, non soltanto non osservano l'astensione dagli hiera, ma assumono comportamenti tali da stravolgere la natura del luogo in cui li compiono, luogo che, proprio in seguito a queste loro azioni, assume la connotazione di bebelos. In questo modo, essi aggiungono ulteriore gravità alla loro violazione / trasgressione (designata dal verbo $\tau \alpha \varrho \alpha \beta \alpha$ ívยเv): quest'ultima, senza dubbio, trae origine da un mancato rispetto degli hiera, ma si qualifica come particolarmente grave per il fatto di spingersi fino a sconfinare in un ambito che agli hiera - questo è l'evidente messaggio - si oppone: غ̇v $\beta \varepsilon \beta \eta \dot{\lambda} \lambda \omega$.

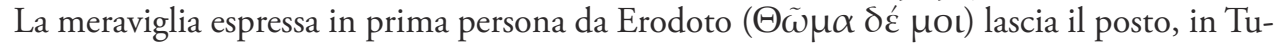
cidide, alla modalità doppiamente indiretta di riportare le parole dell'araldo il quale, a sua vol-

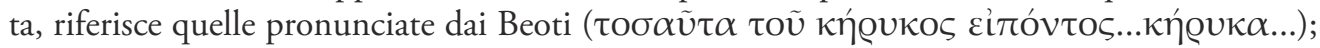
il dato comunque da trattenere, in entrambi i casi, è che la distorsione di norme comportamentali relative agli hiera venga espressa e sottolineata ricorrendo, ancora una volta, alla nozione evocata da bebelos.

La produttività di questa medesima opposizione (bebelos/hieros) agisce anche in contesti dove la qualità di bebelos è attribuita a persone.

Nel Corpus Hippocraticum ${ }^{14}$, il contrasto tra hieros e bebelos è finalizzato a modulare la distinzione tra due categorie di persone, gli hieroi, da un lato, e, dall'altro, i bebeloi, la cui reciproca distanza si misura proprio per il tramite di un differenziato rapporto con la conoscenza degli hiera.

\section{Bebelos $e$ hosios}

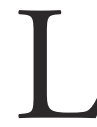

a ricorrenza dell'opposizione di bebelos rispetto a hieros e, più in particolare, la produttività di una coppia complementare quale hiera kai bebela (che abbiamo visto agire per Jesempio nell'epigrafe di Cirene), sembra fare di bebelos un vero e proprio concorrente di hosios $^{15}$, che pure si oppone, e insieme si associa, a hieros secondo le stesse dinamiche formali (si pensi all'espressione hiera kai hosia) ${ }^{16}$. Bebelos si rivela concorrente di hosios anche in ragione del fatto che, al pari di quest'ultimo, esprime, marcatamente, una prospettiva umana rispetto al sacro: è, infatti, in rapporto ad un'attitudine degli uomini che bebelos si definisce, proprio come hosios. Ma l'intersezione tra la nozione di "profano" e quella di bebelos non è sovrapponibile a quella tra il cosiddetto "profano" e hosios: una serie di aspetti denota bebelos rispetto a hosios e mostra il diverso significato che assume, nei due casi, la comune connotazione rispetto a hieros.

13 Sull'uso del termine bebelos in Tucidide e sulla sovrapposizione che in esso si verifica tra accezione "territoriale e spaziale”, da un lato, e “impurità rituale”, dall'altro, cf. Schirripa 2015, 32.

14 Hipp. Lex 5, 2

15 Un caso specifico di equivalenza tra hosios e bebelos, in riferimento alla Lisistrata di Aristofane (vv. 742-43) è analizzato da PeELs 2016, 222-225.

16 Sulla polisemia del termine hosios e sulle relazioni che esso instaura con altri significanti legati alla sfera del sacro, quali katharos, hagnos, dikaios, eusebes, hieros, cf. PEels 2016 (sulla coppia hiera kai hosia cf. in particolare 225230; per un'analisi del dibattito intorno alla nozione di hosios cf. 1-10; sull'ambivalenza e sulla tensione derivante dal fatto che hosios si trovi usato sia come sinonimo di "sacro" che come sinonimo di "profano" cf. 207-251. 
L'etimologia che fa individuare da bebelos il luogo "dove è possibile porre il piede"17, può essere vera o presunta, ma numerosi contesti mostrano come, nella sostanza, la nozione che questo significante richiama tocchi molto da vicino il problema della condivisione dello spazio tra uomini e divinità; bebelos è spesso evocato a definire lo spazio sacro dal punto di vista degli uomini e, in un modo che scopriremo essere peculiare, dei loro movimenti rispetto ad esso.

I contesti nei quali bebelos viene utilizzato sono specchio del processo con cui lo spazio sacro si definisce, implicando diverse gradazioni di avvicinamento, materiale e concreto, da parte degli uomini.

È questo, ad esempio, il nodo intorno al quale Sofocle intreccia l'inizio dell'Edipo a Colono. $(\text { Soph. } O C, 10)^{18}$. Il primo scambio tra Antigone e il padre coinvolge, da subito, la necessità e la volontà di individuare un luogo fisico, connotato rispetto alla nozione di sacro, nel quale il vecchio Edipo possa fermarsi e mettersi seduto. Dato un simile contesto, assume rilievo il fatto che uno dei luoghi, rispetto a cui l'anziano padre chiede di poter trovare collocazione e sedersi

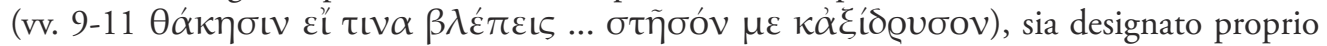

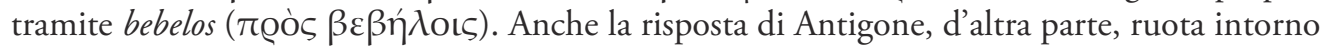
alla circoscrizione di uno spazio sacro, che si compie in rapporto alle esigenze e alle facoltà di movimento di Edipo: la figlia esorta il vecchio a piegare le proprie membra su di una pietra, entro uno spazio la cui sacralità (v. $16 \chi \tilde{\omega} \varrho o \varsigma$ ' 'oo $\delta$ ' i@ós) appare chiaramente comprovata

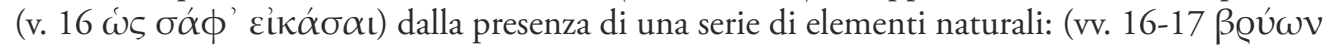

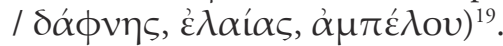

Più avanti, sarà lo straniero ad intervenire per correggere la collocazione di Edipo ancora una volta rispetto allo spazio sacro, intimandogli di allontanarsi dal seggio che sta occupando, designato come luogo che non è lecito calcare (v. $37 \chi \tilde{\omega} \varrho 0 \nu$ oủ $\chi \alpha \hat{\gamma} \gamma$ vòv $\tau \alpha \tau \varepsilon \tilde{\imath} \nu$ ), e che

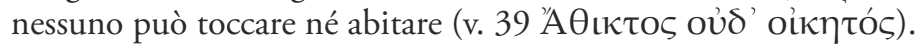

Nella parodo, sarà infine il coro ad invitare con insistenza il vecchio Edipo ad allontanarsi, a tornare indietro (v. 162) e a stare lontano (v. 169); ad uscire da un luogo nel quale non si può andare (v. 166).

Si delinea, per questa via, una rete complessa di indicazioni relative ad azioni che a Edipo è concesso, intimato oppure vietato di compiere nello spazio; esse definiscono e costruiscono l'identità di Edipo ${ }^{20} \mathrm{e}$, insieme, quella dello spazio sacro rispetto al quale egli si muove. È all'interno di un simile sistema, che tramite bebelos prende senso un sistema generativo, al contempo, della sacralità dello spazio e della identità del soggetto; e non solo nel caso specifico dell'Edipo a Colono.

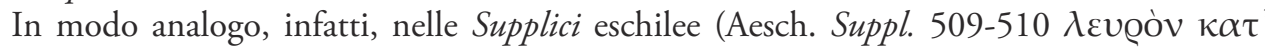

17 Cf. Chantraine 1968, s.v. bebelos. (cfr. anche s.v. baino). Sull'etimologia si veda anche Beeks 2010 sul nesso tra bebelos e la nozione di suolo calpestabile cf. Dion. Hal. AR VII, 8, 4, 2 su cui si veda Schirripa 2015, 136.

18 Sulle modalità con cui alcuni elementi della storia leggendaria di Atene e alcuni aspetti del suo territorio sono trasferiti nelle descrizioni dello spazio presenti dell'Edipo a Colono, cf. Rodighiero 2012. Sull'uso di $\beta \varepsilon ́ \beta \eta \lambda o \varsigma$ nel passo sofocleo cfr. già le osservazioni di Jebb (1885) a.l. Più in generale, sul modo in cui Sofocle si sofferma sulle relazioni tra i suoi personaggi e lo spazio sacro cf. Rенм 2012, 413-415.

19 G. Guidorizzi contrappone una "geografia rituale", percepita da Edipo, a una "geografia reale" presa invece in considerazione, secondo categorie più oggettive, da Antigone (Guidorizzi 2008, 204-205).

20 In tutta la tragedia sofoclea è molto stretto il legame tra il definirsi progressivo di un luogo e la determinazione di un'identità marginale per il vecchio Edipo, come mostra CURSARU 2014, in particolare 119-122, con riferimento ai vv. 9-18. 


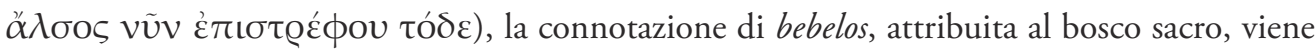
evocata nel contesto di una serie di indicazioni, rivolte dal re Pelasgo al coro delle Danaidi, circa le azioni e gli spostamenti da compiere rispetto ad uno spazio sacro (v. $508 \lambda \varepsilon v \varrho o ̀ v ~ \kappa \alpha \tau$ '

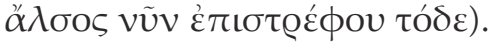

Non pare insomma esservi ragione di dubitare dell'esistenza di un legame privilegiato tra la nozione espressa da bebelos e contesti relativi ad azioni e spostamenti degli uomini rispetto ad uno spazio sacro. La narrazione di Erodoto sui Persiani caduti in battaglia (Hdt. IX, 65, 2), già presa in esame, fa esplicito riferimento alla questione del "fare ingresso" o meno nel recinto sacro

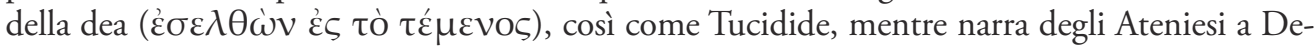

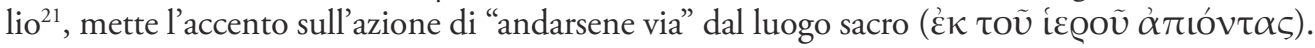

Qualche secolo più tardi, Pausania $(X, 32,17,5)$ applicherà la qualificazione di bebelos ad una persona, con una serie di peculiarità che considereremo più avanti. Quello che ora ci interessa notare è come anche il periegeta si trovi ad incastonare tale designazione in una fitta

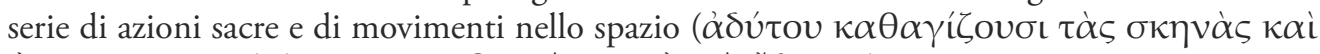

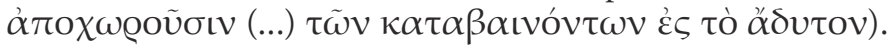

La posizione occupata da bebelos rispetto all'insieme eterogeneo e plurale degli hiera non è, dunque, di semplice opposizione né di meccanica complementarietà. Questa nozione esprime, piuttosto, un aspetto fondativo - seppure parziale - del modo greco di pensare le relazioni degli uomini con gli hiera: essa ha rapporto diretto con lo spazio sacro e con le azioni e i movimenti che gli uomini svolgono rispetto ad esso. E non si tratta di un rapporto indefinito né generico.

Dai contesti che possiamo esaminare emerge, infatti, un elemento ulteriore e decisivo: il delicato sistema di movimenti nello spazio, entro il quale la nozione di bebelos si determina, non ha valore neutro ed è anzi portatore di conseguenze molto particolari, che, come subito vedremo, incidono tanto sullo spazio rispetto al quale tali azioni si svolgono quanto sui soggetti che le compiono.

\section{Bebelos e la costruzione dello spazio sacro}

U na testimonianza efficace, e per questo molto nota, della connessione tra l'esecuzione di un movimento organizzato da parte degli uomini, da un lato, e la definizione e creazione di uno spazio, dall'altro, risiede nella polisemia della nozione di choros $^{22}$. Quest'ultima designa, infatti, al tempo stesso, la danza, l'insieme dei soggetti che la compiono, e il luogo fisico nel quale essa viene eseguita ${ }^{23}$.

Ma questo aspetto specifico e importante del pensiero greco, che lega indissolubilmente una prospettiva umana del movimento al processo di identificazione di uno spazio, assume, nel caso dello spazio sacro, connotazioni ancora più particolari. Le tracce dell'incidenza di un punto di vista umano e, più nel concreto, di veri e propri movimenti agiti da parte degli uomini, sulla creazione stessa dello spazio sacro si ritrovano, ben marcate, già ad un mero livello lessicale, e la sintassi di vari contesti contribuisce poi a mostrarcene, in maniera più chiara e completa, le dinamiche.

21 Thuc. IV, 97, 3.4.

22 Su choros cf. Casevitz 1998.

23 Cf. Chantraine 1968 s.v. choros. 
Termini, e nozioni, come temenos o adyton, ad esempio, si riferiscono ad azioni che, compiute o evitate da parte degli uomini, a questi ultimi consentite oppure vietate, creano, di fatto, lo spazio sacro, e ne decidono la gradazione e le proporzioni, per così dire, di sacralità.

Un aspetto essenziale della maniera greca di pensare il sacro, risiede proprio nella facoltà, tutta umana, di definire e di creare lo spazio entro il quale agisce la "potenza" divina, stabilendo, per il tramite di un simile processo, anche l'identità e l'intensità di quest'ultima.

La "potenza" divina prende forma e senso laddove l'uomo la colloca, e si alimenta anche per il tramite di questa collocazione e del rispetto che verso di essa osservano gli uomini: la "potenza" si accresce in maniera proporzionale alla rigidità della delimitazione impostale dagli uomini, e si rarefa progressivamente, a mano a mano che i suoi confini divengono più labili. Muovendosi nello spazio, e determinando regole per questi suoi movimenti, l'uomo crea lo spazio sacro e, insieme a questo, la forza della potenza divina che in esso si identifica.

In quanto prodotto dell'azione umana del ritagliare (temnein), il temenos ${ }^{24}$ delimita una porzione di terreno dedicato a un dio o a un eroe ${ }^{25}$, ed è attraverso questa operazione, che esso diviene il recinto finalizzato a delimitare lo spazio entro il quale la potenza della divinità è maggiormente concentrata. L'azione del ritagliare, compiuta in una prospettiva e con mezzi del tutto umani, ha, come primo effetto, quello di creare un limite, fisico e concreto, tra uno spazio sacro interno al temenos e uno spazio, ad esso esterno, che sacro non è. Questo intervento esplicito e consapevole da parte degli uomini stabilisce e regola, di fatto, una gradazione nella sacralità dello spazio, una gerarchia nella densità di "potenza" presente, che l'uomo determina e alla quale l'uomo sceglie di sottostare secondo regole stabilite.

In un modo analogo, la nozione di adyton conferma la forza di questa componente nell'elaborazione greca degli hiera. Adyton designa il luogo sacro interdetto all'accesso, rispetto al quale l'uomo non può entrare dentro (dyomai) ${ }^{26}$. È l'uomo a rendere, e a mantenere, sacro uno spazio proprio tramite il processo con cui lo sottrae alla propria disponibilità per offrirlo, invece, a quella del dio, o di una figura umana d'eccezione, che diviene tale proprio per la possibilità concessale di non rispettare il divieto ${ }^{27}$.

Vietare o concedere l'accesso a uno spazio rappresenta, pertanto, uno degli atti costitutivi della sacralità di un luogo, e corrisponde, insieme, al rafforzamento della "potenza" cui tale spazio è stato riservato; tutto ciò porta, non da ultimo, alla definizione dell'identità dei diversi soggetti, sulla base della libertà di movimento della quale è dato loro di disporre.

E secondo queste modalità che si stabiliscono diversi gradi della "potenza": il livello di sacralità dello spazio condiziona i movimenti degli uomini rispetto ad esso ma ne è, prima di tutto, il risultato.

Rispetto alla questione che stiamo affrontando, la nozione corrispondente a bebelos svolge un ruolo non secondario. La qualificazione che esso esprime si trova, infatti, applicata ai luoghi $\mathrm{o}$, come vedremo, a soggetti, in diretto rapporto con la possibilità, concessa o negata, di accedere a uno spazio sacro; per questa via, anche bebelos finisce col riflettere una maggiore o minore concentrazione di "potenza" divina presente in un luogo determinato.

24 Su temenos cf. Patera 2010, 541 e Pedley 2005, 57.

25 Cf. Burkert 2003, 198.

26 Cf. Chantraine 1968, s.v. dyo.

27 Cf. BurKert 2003, 486 e Ustinova 2009, 137-155 (con particolare riferimento all'adyton delfico); sul ruolo del rituale per la costituzione di uno spazio sacro cf. Patera 2010 che riprende ed approfondisce le osservazioni di Etienne 1990. 
L'attribuzione della qualifica di bebelos si rivela insomma un fattore decisivo in contesti in cui è importante mettere a fuoco il grado di sacralità di uno spazio. Un caso esemplare è dato, ancora, dall'inizio dell'Edipo a Colono, dove la questione, per il vecchio Edipo, è proprio quella di sondare la sacralità del luogo. Tramite la domanda che rivolge alla propria figlia, con l'esi-

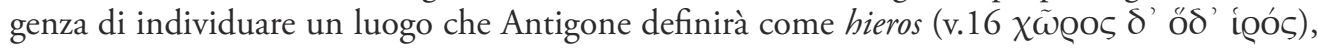
egli distingue tra bebelos ed alsos theon, ponendo le due designazioni in alternanza netta tra loro

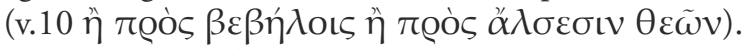

Il dialogo sofocleo sta commisurando la sacralità di diversi spazi, per collocare in essa l'identità del protagonista; ed è significativo che sia proprio la distinzione inconciliabile tra bebelos ed alsos theon ad individuare un primo fondamentale discrimine.

Una relazione di segno opposto tra bebelos ed alsos appare invece quella che si verifica nel passo delle Supplici eschilee dove bebelos, come si è visto, è addirittura attributo di alsos (v. 509

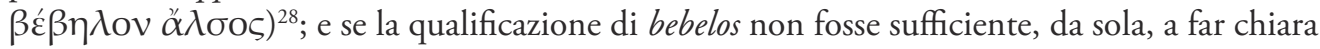
l'accessibilità ${ }^{29}$ dello spazio, le parole che il re Pelasgo ha appena finito di rivolgere al coro delle Danaidi non lasciano dubbi: esse contengono l'imperativo a scendere e ad aggirarsi in questo

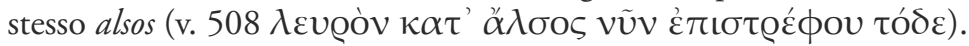

Quella di alsos $^{30}$ è, d'altra parte, a sua volta, una nozione ambigua e problematica, che può designare, oltre ad un bosco sacro (come avviene nel caso di Edipo), un bosco, così e semplicemente, privo di connotazioni ulteriori (a questo sembrerebbe corrispondere l'alsos di Pelasgo), o anche un recinto sacro addirittura sprovvisto di alberi. In ogni caso, a noi interessa il fatto che bebelos intervenga, in modo inequivocabile, a modulare il grado di sacralità di un alsos, nel momento stesso in cui ne rivela il grado di accessibilità o di chiusura.

Ma c'è anche un altro aspetto che non può essere tralasciato. Il bebelon alsos delle Supplici ${ }^{31}$, così privo di limiti fisici che ne compromettano l'accessibilità, sembra perdere, in automatico, quella "potenza" che potrebbe, altrimenti, essere in grado di difendere le Danaidi (v. 509 к $\alpha \grave{i}$

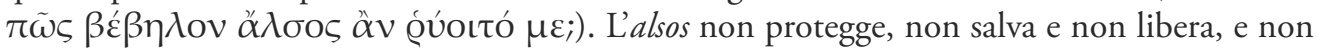
lo fa in ragione del fatto che il luogo in questione è bebelon, è accessibile, e la concentrazione della "potenza", in esso, automaticamente, si rarefa.

Tornando a leggere, a questo punto, la narrazione erodotea relativa ai Persiani che cadono en to bebeloi, fuori dal santuario di Demetra, assume allora un rilievo particolare il commento che lo storico fa seguire alla descrizione dei fatti. Pur ostentando un tradizionale scetticismo circa la propria posizione, e circa la possibilità e la liceità di farsi un'opinione quando si tratti di divinità ( $\varepsilon$ ĺ $^{\prime}$

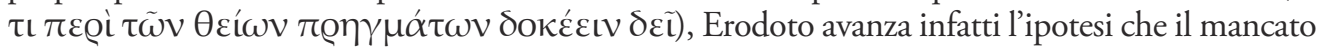
ingresso dei Persiani nel santuario dipenda dalla volontà della dea che non intende accoglierli ( $\eta$

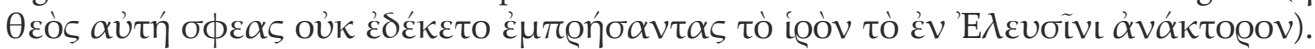

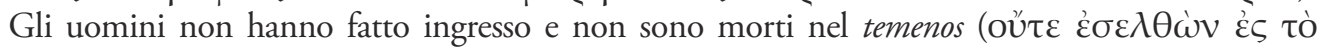

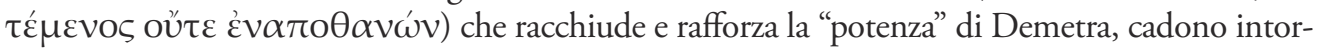

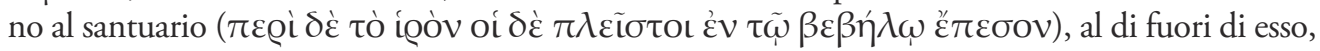

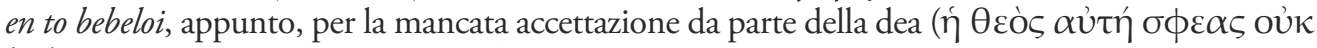
$\dot{\varepsilon} \delta \varepsilon ́ \kappa \varepsilon \tau O)$, atto che costituisce manifestazione, sia pure in negativo, della sua potenza.

28 Cf. Guettel Cole 2004, 63.

29 Si vedano in proposito le osservazioni di Bowen 2013, 252.

30 Su alsos cf. PATERA 2010, 541.

31 La "precisione linguistica" che caratterizza questa scena di hikesia nella tragedia è rilevata da SCHIRRIPA 2015, $114-115$. 
$\mathrm{L}$

a sostanza più profonda della nozione greca di sacro emerge in occasione di processioni, di feste, e di altre situazioni che prevedono il radunarsi di gruppi umani più o meno consistenti. La nozione espressa tramite bebelos regola questa compresenza dal punto di vista fisico: filtra, include o esclude, distribuisce tra gli uomini, secondo meccanismi proporzionali e non omogenei, l'accessibilità allo spazio sacro.

Accanto alla creazione di questo spazio e allo stabilimento di una gamma di gradazioni della potenza divina che in esso risiede, la nozione che sta dietro a bebelos appare intimamente legata anche ad un processo ulteriore, anzi, complementare: essa implica la generazione di gerarchie e di proporzioni differenziate di coinvolgimento e partecipazione degli uomini agli hiera.

Ed una tale implicazione va ben oltre il meccanismo per cui, ad esempio, il superamento di una soglia invalicabile costituisce un atto empio. La morte di Milziade, conseguente, come narra

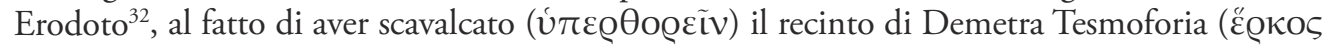

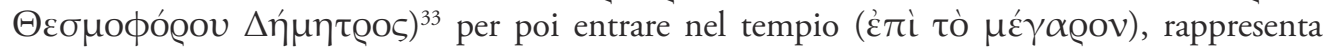
una sorta di caso estremo, in cui il divieto di accesso ad un luogo sacro, la sua trasgressione e la eclatante punizione che ne consegue si corrispondono e si susseguono secondo un meccanismo piuttosto lineare, che poco lascia trasparire del complicato sistema di pensiero che lo sorregge.

Nei contesti in cui è chiamata ad agire la produttività di bebelos, invece, è possibile intravedere una problematicità maggiore, che, al contrario, denuncia per intero la complessità della questione. Insieme a diverse gradazioni di sacralità dello spazio, la nozione espressa da bebelos sembra in qualche modo evocata per gestire, o segnalare, anche una gerarchia di accessibilità al sacro da parte dei diversi soggetti ${ }^{34}$.

La maggiore o minore accessibilità allo spazio sacro che bebelos concorre ad esprimere, determina esclusioni e inclusioni in grado di generare, per i soggetti coinvolti, forme di identità. E tale aspetto può emergere sia nei termini di messa in rilievo, infatti, del singolo soggetto sia, al contrario, di omologazione ad una maggioranza di individui, così come di distinzione tra "iniziati" ed esclusi.

La produttività di bebelos vibra entro contesti in cui la descrizione del rapporto fisico di accesso o di contatto con un luogo sacro viene completata tramite l'uso del pronome di prima persona me: l'equilibrio tra gradazione di sacralità del luogo e criteri di accessibilità mette a

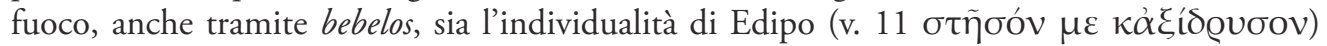
che quella della corifea delle Danaidi (v. 509 àv @úvoıtó $\mu \varepsilon$ ). Mentre, in direzione opposta, vira la gradazione di accesso allo hiron e, specularmente, al bebelon, nel caso dei Persiani di Erodoto: dell'accoglienza della dea risulta privata, indistintamente, la maggior parte di loro (oí $\delta \dot{\varepsilon} \pi \lambda \varepsilon \tilde{\varepsilon} \sigma \tau O \iota)$.

Ma il processo di distinzione gerarchica nella partecipazione e nella contiguità fisica con gli hiera, processo cui la nozione corrispondente a bebelos presiede, si fa evidente, in particolare, qualora l'aggettivo venga attribuito non tanto a luoghi o ad entità astratte, quanto, piuttosto,

32 Hdt VI, 134-135.

33 Anche in questo caso, come già in quello narrato da Thuc. IV, 97, 3-4, le azioni compiute all'interno del luogo sacro non vengono specificate in maniera esplicita ma indicate semplicemente tramite l'espressione ó $\tau \iota$ ঠं

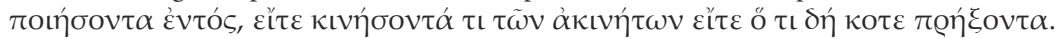

34 Sull'esistenza di una gradation dans la sacralité de la terre che si esprime anche nei règlements concernant la pureté requise pour l'accès aux sanctuaires, cf. PATERA 2010, 544, PARKer 1983, 162 e KeArns 2010, 200-203. 
a persone. Bebeloi divengono, allora, quanti restano esclusi dall'iniziazione a determinati culti, in opposizione alla categoria di quanti vi sono invece ammessi.

L'opposizione, già evocata, che nel Corpus Hippocraticum distingue hieroi anthropoi e bebeloi si sostanzia, in effetti, di una distinta partecipazione agli hiera: la conoscenza di questi ulti-

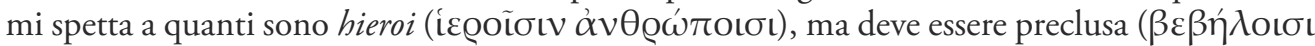
$\left.\delta \varepsilon^{\prime}\right)$ ai bebeloi, prima, almeno, che questi siano stati iniziati agli orgia della conoscenza (T $\alpha \delta \dot{\varepsilon}$

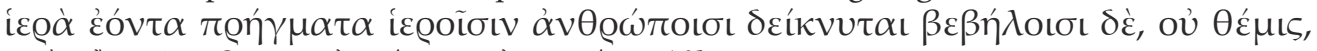

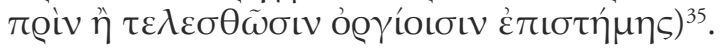

Secondo un meccanismo simile, sia pure in un diverso contesto, Platone, nel Simposio (218b) esclude esplicitamente quanti appartengono alla categoria dei bebeloi dall'insieme di quanti saranno invece ammessi ad ascoltare il racconto di Alcibiade. Bebeloi kai agroikoi si trovano ad es-

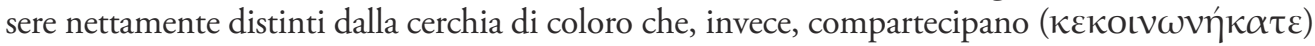
della filosofia, nei suoi aspetti di mania e bakkheia; i primi (bebeloi) sono invitati a chiudere le orecchie, i secondi (agroikoi), al contrario, ad ascoltare. Si tratta dunque di modulare, appoggiandosi anche in questo caso alla designazione di bebeloi, i diversi gradi di accesso a un sapere.

D'altra parte, quando, negli Eraclidi, Euripide ricorre al neutro plurale bebela per riferirsi a un'entità astratta, i logia palaia, la posta in gioco riguarda una distinzione connessa tra ciò che è dato conoscere e ciò che invece deve restare ignoto e nascosto. Bebela è, rispetto a kekrymmena, distinto e complementare, ed esprime, in tal modo, una delle possibili modulazioni nell'accesso dell'uomo alla conoscenza, entro un ambito che coinvolge cantori d'oracoli (v. 403

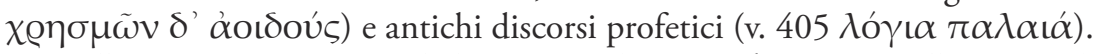

Nell'iscrizione contenente la legge sacra di Cirene ${ }^{36}$, sia pure nell'oscurità del senso da attribuire al contenuto ${ }^{37}$, appare chiaro come l'opposizione tra hagnos e babalos serva a definire la distinzione tra le due categorie di persone ammesse entrambe a partecipare ai riti degli Acamantidi. La designazione di bebelos si trova così coinvolta a delineare l'identità dei soggetti direttamente definiti come tali, ma anche quella di una particolare categoria di morti (hiaroi), partecipando ai culti dei quali non ci si contamina, a differenza di quanto accade per gli altri che rendono bebalos chi, invece, non lo era. Sebbene non sia affatto chiara la natura specifica dei riti in questione, il riferimento di bebelos ad un soggetto umano sembra comunque confermare una tendenza.

Bebelos qualifica, infine, un soggetto umano anche nella descrizione che Pausania ${ }^{38}$ offre della festa celebrata in onore della dea egiziana Iside dagli abitanti di Titorea nella Focide. Il racconto evoca la nozione di bebelos dopo aver restituito un dettagliato resoconto della preparazione della festa ${ }^{39}$ e delle azioni richieste per la sua celebrazione, tra le quali si nota l'attenta precisazione dei criteri di accessibilità al tempio.

La distinzione tra diverse categorie di soggetti avviene per il tramite della descrizione delle diverse azioni che questi hanno incarico di svolgere o divieto di compiere. È in questa serie che

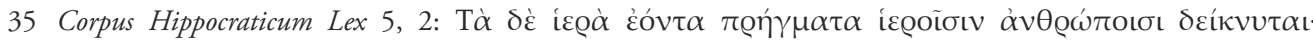

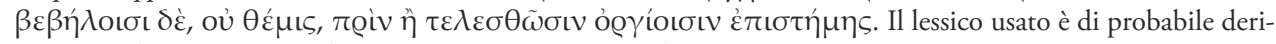
vazione orfico-pitagorica (cf. VegetTi 1976, 418 n.5). Cf. anche Nikolova 1996, 506.

36 Cf. Rodhes, Osborne 2003, 495-505; sul carattere problematico della nozione di "legge sacra" si vedano le riflessioni di Carbon, Pirenne-Delforge 20 i 2.

37 Sulla difficoltà e sul carattere enigmatico di questa iscrizione cf. RoBERTson 2010, 6-10.

38 Paus. X, 32, 17, 5 .

39 All'interno di questa lunga descrizione V. Pirenne-Delforge mette in rilievo la presenza dei verbi thyein e kathaghizein e quella del sostantivo thysia (Pirenne-Delforge 2008, 227, n. 228). 
si inserisce l'individuo designato come bebelos, il quale - viene specificato - non rientra nella

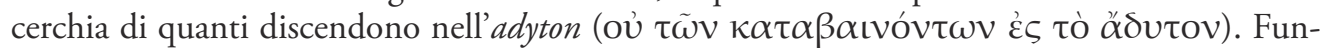
zione e status di un soggetto si trovano ad essere espressi, pertanto, ancora una volta tramite bebelos, nei termini di una gradazione di accessibilità a determinati spazi sacri.

\section{Conclusione}

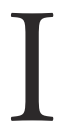
contesti nei quali bebelos appare produttivo non si limitano a confermare il superamento della coppia antinomica "sacro" / "profano", svelandone semplicemente il carattere anacronistico e riduttivo. Mentre sfugge a una collocazione definitiva in seno alla seconda delle due definizioni, quella appunto di "profano", bebelos sembra recare traccia di una serie di dinamiche responsabili, da un lato, di dare origine all'antinomia in questione, dall'altra, al contrario, di impedire a quest'ultima di fissarsi una volta per tutte.

Più che in un'opposizione netta "sacro" / "profano", bebelos appare, in effetti, coinvolto in un gioco molto complesso, all'interno di un sistema di tensioni e di complementarietà assai più ampio di quanto non sia un semplice meccanismo bipolare. Ad essere implicata è, piuttosto, una molteplicità di livelli, orientata secondo una serie di gradazioni di partecipazione agli hiera: una sorta di ripartizione proporzionale della accessibilità al sacro, che coinvolge, in primo luogo, il movimento in rapporto allo spazio. È questo un aspetto essenziale per comprendere il modo greco di pensare il sacro, una delle cui massime espressioni, fisiche e sociali, era, non a caso, la pompe $e^{40}$.

Più in particolare, tramite le dinamiche implicate da bebelos, si definiscono, insieme, da un lato, la potenza della divinità, e, dall'altro, l'identità dell'uomo rispetto agli dei e rispetto agli altri uomini, tanto che, in alcuni casi, bebelos arriva a qualificare non oggetti o spazi ma, direttamente, persone. Nel momento stesso in cui prende forma una sorta di gradazione del carattere sacro dello spazio, si compie un rafforzamento della potenza divina - ingrediente necessario alla costituzione di un luogo di culto - e si regola la partecipazione dell'uomo a questo aspetto del sacro.

Bebelos sembra in tal senso avere a che fare con una declinazione a livello individuale di quello che la pompe costituisce a livello collettivo.

In questo sistema di proporzioni e di equilibri, che la nozione di bebelos contribuisce a mettere in luce, non emergono divieti assoluti e definiti una volta per tutte, ma, piuttosto, una serie molteplice di regole d'accessibilità al sacro e di contiguità con esso, mutevole perché legata alla varietà delle dinamiche rituali, delle gradazioni, di ripartizioni più o meno equilibrate: bebelos si specializza così nel filtrare una modulazione, socialmente e storicamente determinata, di accesso e di partecipazione al sacro.

Lucia Marrucci

Laboratorio di Antropologia del Mondo Antico Dipartimento di Filologia, Letteratura, Linguistica Università di Pisa Via Galvani 56126 Pisa 1.marrucci@flcl.unipi.it 40 Sulla pompe in generale si vedano Larson 2007, 6 e VLASSOPOULOs 2015, 264 ss. 


\section{Bibliografía}

BEEKS 2010

R. Beeks, Etymological Dictionary of Greek, LeidenBoston 2010.

BORgEAUd 1994

$\mathrm{Ph}$. Borgeaud, «Le couple sacré/profane. Genève et fortune d'un concept opératoire en histoire des religions", Revue de l'histoire des religions 211, 4 (1994), $387-418$

BOWEN 2013

A.J. Bowen, Aeschylus' Suppliant Women, Oxford 2013.

\section{BREMMER 1998}

J. N. Bremmer, "Religion' and the Opposition 'Sacred' vs. 'Profane'", in F. Graf (ed.), Ansichten griechischer Rituale, Stuttgart-Leipzig, 1998, 9-32.

BURKERT 2003

W. Burkert, La religione greca (ed. or. Stuttgart 1977), $2^{a}$ ed. it. a cura di G. Arrigoni, Milano 2003.

Carbon, Pirenne Delforge 2012

G.-M- Carbon, V. Pirenne-Delforge, «Beyond Greek Sacred Laws», Kernos 25 (2012), 163-182.

\section{Casevitz 1998}

M. Casevitz, "Remarques sur l'histoire de quelques mots exprimant l'espace en grec», REA 100 (1998), 417-435.

Cursaru 2014

G. Cursaru, "Chora au cœur des enjeux politicoreligieux et de la rhétorique patriotique dans les tragédies grecques. Étude de cas : Edipe à Colone de Sophocles», Mythos 8 (2014), 167-178.

Di Donato 2001

R. Di Donato, Prolegomena ad uno studio storico antropologico della religione greca, Pisa $2001\left(2014^{2}\right)$.

ETIENNE 1990

R. Etienne, "Autel et sacrifices", in Le sanctuaire grec, Entretiens Fondation Hardt, Genève 1990, 291-312.

Guettel Cole 2004

S. Guettel Cole, Landscape, Gender, and Ritual Space: the Ancient Greek Experience, Berkeley 2004.

Guidorizzi 2008

Sofocle, Edipo a Colono, a cura di G. Avezzù e G. Guidorizzi, traduzione di G. Cerri, Milano 2008.

JEFFREY 1961

L.H. Jeffrey, Local Scripts of Archaic Greece, Oxford 1961.
LARSON 2007

J. Larson, Ancient Greek Cults, New York 2007.

Nikolova 1996

V. Nikolova, «A Hypothesis on dichotomic essence of the Hippocratic doctrine», in N. Wittern, P. Pellegrin (eds), Medizin der Antike, Bd.1, Hippokratische Medizin und antike Philosophie, Hildesheim-Zurich-New York 1996, 491-512.

PARKer 1983

R. Parker, Miasma. Pollution and Purification in Early Greek Religion, Oxford 1983

PARKer 2005

R. Parker, Polytheism and Society at Athens, Oxford 2005.

PARKer 2011

R. Parker, On Greek Religion, Cornell 2011.

PATERa 2010

J. Patera, «Espace et structures cultuels du sanctuaire grec : la construction du vocabulaire», Revue de l'histoire des religions 4 (2010), 535-551.

Pedley 2005

J. Pedley Sanctuaries and the Sacred in the Ancient Greek World, Cambridge 2005.

Peels 2016

S. Peels, Hosios. A Semantic Study of Greek Piety, Leiden-Boston 2016.

Pirenne Delforge 2008

V. Pirenne Delforge, Retour à la source. Pausanias et la religion grecque, Liège 2008.

ReHM 2012

R. Rehm, "Ritual in Sophocles», in A. Markantonatos (ed.), Brill's Companion to Sophocles, LeidenBoston 2012, 411-427.

Rhodes, Osborne 2003

P. J. Rhodes, R. Osborne, Greek Hiostorical Inscriptions 404-323 BC, Oxford 2003.

ROBERTSON 2010

N. Robertson, Religion and Reconciliation in Greek Cities. The Sacred Laws of Selinus and Cyrene, Oxford 2010.

RODIGHIERO 2012

A. Rodighiero, "The Sense of Place», in A. Markantonatos, B. Zimmermann (eds), Crisis on Stage. Tragedy and Comedy in Late Fifth-Century Athens, BerlinBoston 2012, 55-80. 
SCHIRRIPA 2015

P. Schirripa, Il tempio, il rituale, il giuramento. Spazi del sacro in Tucidide, Roma 2015.

UsTINOVA 2009

Y. Ustinova, Caves and the Ancient Greek Mind. Descending Underground in the Search for Ultimate Truth, Oxford 2009.

VegetTTI 1976

Ippocrate, Opere, a cura di M. Vegetti, Torino $1976^{2}$ (1965).

\section{VERNANT 1965}

J.-P. Vernant, Mythe et pensée chez les Grecs, Paris 1965.

\section{VERNANT 1975}

J.-P. Vernant, Religion grecque, religions antiques, leçon inaugurale de la chaire d'études comparées des religions antiques, Collège de France, 5.12.1975 (= J.-P. Vernant, Religions, histoires, raisons, Paris, 1979, 5-34).
VERnANT 1990

J.-P. Vernant, Mythe et religion en Grèce ancienne, Paris 1990 , tr. it. a cura di R. Di Donato, Roma 2003.

VERNANT 1995

J.-P. Vernant, Passé et Présent. Contributions à une psychologie historique réunies par Riccardo Di Donato, Roma, 1995.

Vignolo Munson 2001

R. Vignolo Munson, Telling Wonders. Ethnographic and Political Discourse in the Work of Herodotus, Ann Arbor 2001.

VLASSOPOULOS 2015

K. Vlassopouls, "Religion in communities», in J. Kindt, E. Eidinow (eds), The Oxford Handbook of Ancient Greek Religion, Oxford 2015, 257-272. 